\title{
LXVI. Observations on a paper by Prof. Faraday concerning electric conduction and the nature of matter
}

\section{Richard Laming Esq.}

To cite this article: Richard Laming Esq. (1845) LXVI. Observations on a paper by Prof. Faraday concerning electric conduction and the nature of matter, Philosophical Magazine Series 3 , 27:182, 420-423, DOI: $10.1080 / 14786444508646245$

To link to this article: http://dx.doi.org/10.1080/14786444508646245

冓 Published online: 30 Apr 2009.

Submit your article to this journal $\pi$

ЏII Article views: 4

Q View related articles $\sqsubset$ 
LXVI. Observations on a paper by Prof. Faraday concerning Electric Conduction and the Nature of Matter. By Richand LaMing, Esq.

\section{To Richard Taylor, Esq.}

Sir,

IN February of last year a letter from Professor Faraday was published by you, expressing an opinion that the immaterial centres of force of Boscovich $*$ have a greater claim to be regarded as true than the solid atoms of Newton; and in which he represents his preference to result from the contemplation of certain facts relating to electrical conduction and insulation. In going over his arguments I have not arrived at the same conclusion, owing to a difficulty in admitting an assumption which I find mixed up with the facts. It will be better to give Mr.Faraday's meaning in his own words: he says, "The view of the atomic constitution of matter which I think is most prevalent, is that which considers the atom as something material having a certain volume, upon which those powers were impressed at the creation, which have given it, from that time to the present, the capability of constituting, when many atoms are congregated together into groups, the different substances whose effects and properties we observe. These, though grouped and held together by their powers, do not touch each other, but have intervening space, otherwise pressure or cold could not make a body contract into a smaller bulk, nor heat or tension make it larger; in liquids these atoms or particles are free to move about one another, and in vapours or gases they are also present, but removed very much further apart, though still related to each other by their powers..... If the view of the constitution of matter already referred to be assumed to be correct, and $I$ may be allowed to speak of the particles of matter and of the space between them as two different things, then space must be taken as the only continuous part, for the particles are considered as separated by space from each other." All this may be accepted as descriptive of matter as it is regarded by the theory of solidity; and with this admission we proceed at once to the main question, namely, to which of the two parts of a body does its electrical conducting property belong; does it appertain to the centres of force themselves, or to the spaces which envelope them? I agree with Mr. Faraday that the conducting property does not belong to space, because if it did, it would follow that as all bodies indiscriminately are re-

[" See Priestley's "History of Vision," \&c., and "Disquisitions on Matter and Spirit," vol. i. p. 34, \&c.-ED.] 
plete with spaces, so all bodies, without exception, would be conductors, however much their natures might otherwise differ. This conclusion is manifestly inevitable, for to deny it would be to impute different.qualities to different parts of space. Not so inevitable, however, is the next conclusion which I have to notice: "Metal is a conductor ; but," says Mr. Faraday, "how can this be except space be a conductor? for it is the only continuous part of the metal ; space therefore (holding the theory of solid atoms) must be a conductor, or else the metals could not conduct." That gentleman therefore sees no alternative but in believing that "s the reasoning ends in a subversion of the theory (of solid atoms) altogether."

This second conclusion would be as unavoidable as the preceding one, provided we knew, as a fact, that absolute continuity is necessary to electrical conduction; Mr. Faraday has assumed this to be the case, but I am not aware that anything has ever been observed in the nature of matter from which it can be inferred. By the old maxim, that matter cannot act where it is not, absolute continuity is made necessary to conduction ; but this maxim is itself an assumption needing the evidence of facts, and therefore it may not be adduced as a proof of any other assumption.

The chief cause why I have thought it necessary thus to attempt the vindication of the theory of atoms from a charge of inconsistency, is because I think there is a way of looking at that theory, by which the conducting and insulating properties of bodies appear more intelligible than on other doctrines; and this without involving any other assumption (beyond the mere existence of the atoms) than the hypothesis that different sorts of atoms are naturally associated with unequal quantities of electricity. The degree of probability attaching to this hypothesis may be estimated by reflecting that if we had to assume its untruthfulness, that is to say, that all atoms, however unlike in their natures, had equal quantities of electricity naturally combined with them, the assumption might be deemed much more questionable. I will now beg permission to give an outline of the view I have taken of the theory of atoms, so far as it relates to electrical conduction and insulation; and as it is simple, it may be done in a few words.

In his "Researches," Mr. Faraday has adduced an experiment to prove that the quantity of electricity naturally combined with matter is enormously great; after making every allowance for the difficulty of a correct estimation, we may safely infer the quantity to be great enough to apportion to 
each atom of matter sufficient electricity to envelope it. In the second place, we know, by facts, that the force by which electricity is attracted by matter is greater at shorter distances; from whence it follows that the electricity around an atom of matter will approach to it as near as possible, thus forming a sphere of which the atom is the centre. Again, by the theory of solidity, a mass of electrical matter, or electricity, may be regarded as composed of electrical atoms, just as a mass of ordinary matter contains ordinary atoms; and thus the sphere of electricity which surrounds an ordinary atom will consist of a number of electrical atoms arranged in concentric strata. The number of electrical atoms belonging to a given ordinary atom may be assumed to be such as to complete its external spherical stratum, or, on the contrary, it may be such as to leave that external spherical stratum more or less imperfect. Now, in the former of these two cases, where the sphere of electricity has an unbroken surface, we have all the conditions necessary for electrical insulation; and in the latter case, where the surface of the sphere of electricity is broken, we have all the conditions essential to electrical conduction.

To explain this more fully we may begin with conductors. Imagine a line of ordinary atoms, each of which naturally has on the bounding surface of its mass of electricity ten electrical stoms; and place the ends of this line in connexion with two bodies oppositely electrified. Thereupon so many of the electrical atoms of the plus charge as can find room will place themselves in the broken electrical stratum of the first ordinary atom of the conductor; because when there a certain part of each electrical atom then in that stratum, in the aggregate amounting to ten whole atoms, will be attracted by the said ordinary atom. This manifestly is required by the law of force, which is greater at the lesser distance; and because the aggregate of all the parts is at a less distance than are any ten whole atoms in the same stratum. At the other end of the conducting line the negative body will be appropriating to itself the ten superficial electrical atoms naturally belonging to the most proximate ordinary atom of the conductor; because this atom, in its turn, can make an equal demand upon the next in succession, that one on its neighbour, and so onward continually to the plus end of the line, without at any time the attractive force being called upon to act at an increased distance. The first ten plus atoms being thus virtually discharged into the minus body, a second similar number will then be transferred in like manner, and after that a third, and thus the conducting action will be repeated as many times as is requisite to convey the whole electrical 
charge of the plus body into that which was equally minus. According to this explanation of electrical conduction, that phænomenon essentially depends on the facility possessed by an ordinary atom to receive on the surface of its mass of electricity a surplus of electrical atoms at an equal distance from the common centre, as are already some of its own.

In the case of insulators, we have ordinary atoms with perfect external spherical strata; a condition obviously incapable of conducting electricity, because no surplus number of electrical atoms, presented by a plus body, can possibly arrive so near to the first ordinary atom of a line as are already its own most distant electrical atoms.

It is not my intention, Sir, to enlarge here on this view of matter and of electrical action, because to trace the branches of the subject would exceed the limits that could be allowed in your valuable periodical. I hope soon to lay before the public my electrical opinions in a detailed form; but, before concluding my letter, I will just allude to what appears to me to be an aptitude in the theory of atoms to meet the exigences of electrical excitation and chenical affinity; which I think may both be shown to proceed from one and the same cause, the difference in result depending only upon unequal susceptibilities to locomotion. 'To have the most simple case, imagine two dissimilar ordinary atoms to be placed in contiguity; the atoms, being dissimilar, have unequal quantities of electricity around them, forming spheres of unequal radii. On the surface of each electrical sphere let there be one electrical atom, necessary to make up the complement of its mass of electricity. It is manifest that, on the reciprocal approach of the electrical spheres, the two external electrical atoms will place themselves between them, both in a line at right angles to the common axis, and in this position one-half of each external electrical atom be attracted towards each ordinary atom. If the ordinary atoms be now forcibly separated, both of the external electrical atoms will attach themselves to the sphere of least radius; because the electrical attraction acts with the greater force at the lesser distance, and because also an atom is by its nature indivisible.

I am, Sir, yours, \&c., Richard Laming.

Clichy-la-Garenne, near Paris, Oct. 4, 1845. 